\title{
Unconditioned stimulus intensity and asymptotic performance in human eyelid conditioning '
}

WLLLIAM F. PROKASY

UNIVERSITY OF UTAH

It is shown that whether or not the asymptotic performance of high-rate conditioners in human eyelid conditioning is affected by unconditioned stimulus intensity depends upon the conditioning context, while the acquisition performance of low-rate conditioners seems to be a function of unconditioned stimulus intensity regardless of conditioning context.

\section{Problem and Method}

Burstein (1965) concluded that differences in asymptotic performance as a function of unconditioned stimulus intensity (UCSI) were attributable to the greater number of non-conditioners (NCs) in the low UCSI groups rather than to performance differences between conditioners (Cs). Despite minor objections (Burstein, 1967), Spence \& Platt (1966) have shown that not all performance differences as a function of UCSI are attributable to differences in the number of NCs. However, since Spence and Platt conducted their comparisons on data from studies in which asymptotic performance was not assured, they failed to come to grips with Burstein's conclusion that, given Cs, asymptotic performance is not affected by UCSI.

The purpose of this note is to report relevant data from a study in which asymptotic performance was approximated and In which overall differences as a function of UCSI were obtained. Since the study was reported elsewhere (Prokasy \& Allen, 1966), the entire procedure will not be detailed. It was a 2 by 2 factorial design with two levels of UCSI (100 vs. $200 \mathrm{~mm} \mathrm{Hg}$ ) and the standard conditioning procedure versus the masiding procedure reported by Spence, Homzie, \& Rutledge (1964). All $60 \mathrm{Ss}$ in each of the four groups recelved 10 adaptation trials followed by 300 paired trials. The CS was a 1000-cps tone and the interstimulus interval was 500 msec. Group designations are: HN for high intensity, normal procedure; LN for low intensity, normal procedure; HM for high intensity, masking procedure; and LM for low intensity, masking procedure.

Results

The first task was to define the Cs and NCs. Four definitions of a NC were examined: (1) response probability of .2 or less for the first 40 trials; (2) response probability of .2 or less for the first 60 trials; (3) response probability of .1 or less for the first 40 trials; and (4) response probability of .1 or less for the first 60 trials. All definitions yielded identical data trends. Definition (1) was finally employed because it defined the largest number of $\mathrm{NCs}$, and hence, yielded greater reliability for statistical purposes.
Table 1. Response Probability Across Three Blocks of Trials

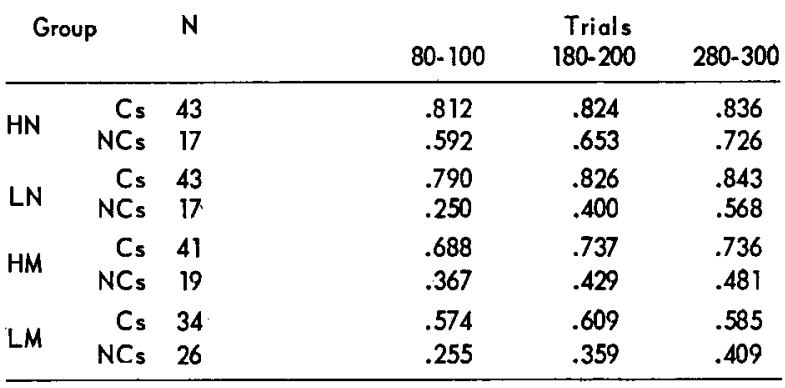

The data of primary interest are summarized in Table 1. The $\mathrm{Ss}$ in each of the four treatment combinations have been classified as $\mathrm{Cs}$ or $\mathrm{NCs}$, and response probability is reported for the eight subsets of Ss for Trials 80-100, 180-200, and 280-300. Analyses of variance were conducted separately for normal and masking groups, and separately for the Cs and NCs within these groups, and are summarized in Table 2.

Regardless of classification, the Ss originally defined as NCs did not remain that way: The increase in response probablltty shown by the NCs across trial blocks is reliable. As a further check on the reliability of this increase, probabllity was found to be .2 or less over the last 150 trials for only one, two, three, and seven NCs, respectively, in Groups $H N, L N, H M$, and LM.

Increases in response probabllity beyond the 100th trial shown by the Cs were slight and not reliable. Since the comparisons spanned 200 trials, it would appear that asymptote had been reasonably well approximated.

The effects of UCSI were not uniform across groups. In the normal conditioning context, the $\mathrm{Cs}$ did not differ in asymptotic behavior as a function of UCSI. In contrast, the higher UCSI resulted in

Table 2. Analyses of Variance of Response Probability

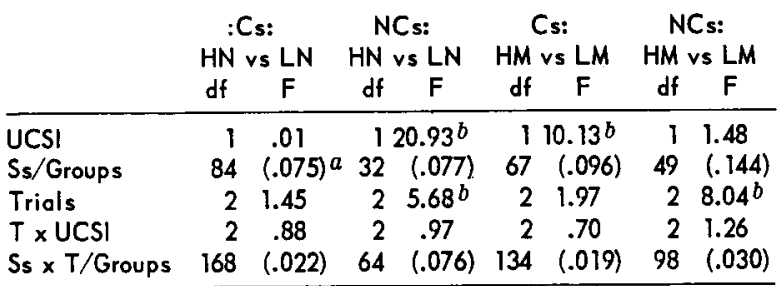

a Error mean squares are in parentheses

b $p<.05$ 
greater asymptotic response probability in the Cs trained with the masking condition. In both the normal and masking procedures, the $\mathrm{NCs}$ receiving the higher UCSI yielded a higher response probability than did the NCs receiving the lower UCSI, though this effect was reliable only with the normal procedurre. It should be noted that these latter effects are on acquisition, not asymptotic, performance.

Discussion

It is entirely possible that different UCSIs result in different numbers of Ss who condition, and that the effect of non-conditioners is to exaggerate group differences as a function of UCSI. In this study, however, two facts are apparent: Ss originally defined as NCs did not remain non-conditioners, and UCSI differentially affected the performance of Ss who conditioned. Therefore, while the influence of NCs may exist in past studies, UCSI effects observed in this study cannot be explained by the failure of some Ss to condition. The data of this study suggest that Ss separated as $\mathrm{NCs}$ by a performance criterion early in training are likely to be low-rate conditioners rather than non-conditioners.

Burstein's specific suggestion was that asymptotic performance is independent of UCSI. For Ss conditioned with the normal conditioning procedure, this study permits the conclusion that high-rate conditioners (1.e., $\mathrm{Cs}$ who have reached asymptote by the 100th trial) are not differentially affected by UCSI at asymptote. The acquisition rate of low-rate conditioners is differentially affected by UCSI, though it remains to be seen whether a difference exists at asymptote. A general statement to the effect that the asymptotic performance of $\mathrm{Cs}$ is independent of UCSI cannot be made, however, since there were asymptotic differences when the masking procedure was employed.

It is to be noted, finally, that Spence and Platt err when they imply that the studies they reviewed show that asymptotic performance is differentially affected by UCSI with a conventional conditioning procedure. The differences they reported are real, but may just as easily reflect differences in acquisition rates. This is because the studies they reviewed are generally confined to those with 100 or fewer conditioning trials. The clear evidence (e.g., Prokasy, 1965; Prokasy \& Allen, 1966) of performance increments beyond 100 trials precludes definitive statements about asymptotes based on studies incorporating fewer than 150 or 200 trials. Even in the present study, with 300 trials, it was not evident that the NCs had asymptoted.

\section{References}

Burstein, K. R. The influence of UCS upon the acquisition of the conditioned eyelid response. Psychon. Sci., 1965, 2, 303-304.

Burstein, K. R. UCS intensity and eyelid conditioning: Another look. Psychon. Sci., 1967, 7, 81-821

Prokasy, W. F. Stimulus fluctuation, reactive inhibition, and time between trials in classical eyelid conditioning. J. exp. P sychol., $1965,70,464-472$.

Prokasy, W. F., \& Allen, C. K. Drive times habit in human eyelid conditioning. Psychon. Sci., 1966, 5, 155-156.

Spence, K. W., Homzie, M. J., \& Rutledge, E. F. Extinction of the human eyelid $C R$ as a function of the discriminability of the change from acquisition to extinction. J. exp. Psychol., 1964, 67, 545-552.

Spence, K. W., \& Platt, J. R. UCS intensity and performance in eyelid conditioning. Psychol. Bull., 1966, 65, 1-10.

\section{Note}

1. This research was supported by NSF Grants G-18119 and GB 5627. I would like to acknowledge the technical assistance of Karol Kumpfer and Reginald Swartz. 